\title{
AN ANALOGUE OF THE ERDÔS-KAC THEOREM FOR THE SPECIAL LINEAR GROUP OVER THE INTEGERS
}

\author{
DANIEL EL-BAZ
}

\begin{abstract}
We investigate the number of prime factors of individual entries for matrices in the special linear group over the integers. We show that, when properly normalised, it satisfies a central limit theorem of Erdős-Kac-type. To do so, we employ a sieve-theoretic set-up due to Granville and Soundararajan. We also make use of an estimate coming from homogeneous dynamics due to Gorodnik and Nevo.
\end{abstract}

\section{INTRODUCTION}

The celebrated Erdôs-Kac theorem is a central limit theorem for the number of (distinct) prime factors $\omega$ of a "random" integer, in the following sense: for every $x \in \mathbb{R}$, we have

$$
\lim _{n \rightarrow+\infty} \frac{1}{n} \#\left\{1 \leq m \leq n: \frac{\omega(m)-\log \log n}{\sqrt{\log \log n}} \leq x\right\}=\frac{1}{\sqrt{2 \pi}} \int_{-\infty}^{x} e^{-\frac{t^{2}}{2}} d t .
$$

There is an abundant number of results of this type for the number of prime factors of various sequences of integers: shifted primes [8], values of integer polynomials [9] and friable numbers [10, 1, 11, to cite but a few examples.

In this note, we study the number of prime factors of the entries in integer matrices of unit determinant. More precisely, we define, for $n \geq 2$ and $T>0$,

$$
V_{T}(\mathbb{Z})=\left\{g \in \mathrm{SL}_{n}(\mathbb{Z}):\|g\| \leq T\right\},
$$

where for $g \in \mathrm{SL}_{n}(\mathbb{Z}),\|g\|=\sqrt{\operatorname{Tr}\left(g^{\mathrm{t}} g\right)}$ is the Frobenius norm.

For an integer $n \geq 1$, we let $\omega(n)$ be the number of distinct prime factors of $n$. We extend $\omega$ to $\mathbb{Z}$ by defining $\omega(0)=0$ and for $n \geq 1, \omega(-n)=\omega(n)$. Our main theorem can now be stated.

Theorem 1.1. For every $n \geq 2$, every $x \in \mathbb{R}$ and for each pair $(i, j) \in\{1, \ldots, n\}^{2}$, we have

$$
\lim _{T \rightarrow+\infty} \frac{1}{\# V_{T}(\mathbb{Z})} \#\left\{g \in V_{T}(\mathbb{Z}): \frac{\omega\left(g_{i, j}\right)-\log \log T}{\sqrt{\log \log T}} \leq x\right\}=\frac{1}{\sqrt{2 \pi}} \int_{-\infty}^{x} e^{-\frac{t^{2}}{2}} d t .
$$

Our proof uses the sieve-theoretic framework unravelled by Granville and Soundararajan in 2006 [7, which we recall in subsection 3.1] so as to be self-contained. To obtain the necessary estimates to feed into the sieve, we apply a deep result of Gorodnik and Nevo from 2010 [6].

We note that since this effective congruence counting is the key input, one can also apply such arguments to thin linear groups, thanks to the work of Bourgain, Gamburd and Sarnak 3. Indeed, this was done in the case of Apollonian circle packings with integral curvatures by Djanković in 4 .

Acknowledgements: I would like to thank Efthymios Sofos for asking the question answered in this paper. I am grateful to Zeev Rudnick for several helpful conversations and encouraging me to write up this short note. I am also grateful to Carlo Pagano for the comments he made on a previous version of this draft that led to an improved presentation. This research was supported by the European Research Council under

2010 Mathematics Subject Classification. Primary 11N36; Secondary 22F30.

Key words and phrases. sieve theory, probabilistic number theory, effective congruence counting. 
the European Union's Seventh Framework Programme (FP7/2007-2013) / ERC grant agreement $\mathrm{n}^{\circ} 320755$.

\section{Counting With COngruences}

We need the following special case of [6, Corollary 5.2].

For $n \geq 2$ and a positive integer $Q$, define the following - principal congruence subgroup of $\mathrm{SL}_{n}(\mathbb{Z})$ :

$$
\Gamma(Q)=\left\{g \in \mathrm{SL}_{n}(\mathbb{Z}): g \equiv I_{n} \quad(\bmod Q)\right\} .
$$

Claim 2.1 (Gorodnik-Nevo). For every $n \geq 2$, there exists $\delta>0$ such that for every $M \in \mathrm{SL}_{n}(\mathbb{Z})$ and every integer $Q \geq 1$,

$$
\#\{A \in \Gamma(Q) M:\|A\| \leq T\}=\frac{\# V_{T}(\mathbb{Z})}{\left[\mathrm{SL}_{n}(\mathbb{Z}): \Gamma(Q)\right]}+O\left(\# V_{T}(\mathbb{Z})^{1-\delta}\right),
$$

where the implied constant is independent of $Q$.

In fact, we shall require the following consequence.

Corollary 2.1. For every $n \geq 2$, there exists $\delta>0$ such that for every square-free integer $q$ and every $(i, j) \in\{1, \ldots, n\}^{2}$,

$$
\#\left\{g \in V_{T}(\mathbb{Z}): q \mid g_{i, j}\right\}=\prod_{\substack{p \in \mathbb{P} \\ p \mid q}} \frac{p^{n-1}-1}{p^{n}-1} \# V_{T}(\mathbb{Z})+O\left(q^{n^{2}-2} \# V_{T}(\mathbb{Z})^{1-\delta}\right)
$$

Proof. Fix $n \geq 2$ and $(i, j) \in\{1, \ldots, n\}^{2}$.

The group $\Gamma(q)$ acts by left multiplication on the set $\left\{g \in \mathrm{SL}_{n}(\mathbb{Z}): q \mid g_{i, j}\right\}$, which we may therefore view as a disjoint union of finitely (a priori, at most $q^{n^{2}-1}$ ) $\Gamma(q)$-orbits. We restrict the orbits to elements having norm at most $T$ and note that each orbit has the same number of points, given by the formula in Claim 2.1.

$$
\frac{\# V_{T}(\mathbb{Z})}{\left[\mathrm{SL}_{n}(\mathbb{Z}): \Gamma(q)\right]}+O\left(\# V_{T}(\mathbb{Z})^{1-\delta}\right)
$$

First, $\mathrm{SL}_{n}(\mathbb{Z}) / \Gamma(q) \cong \mathrm{SL}_{n}(\mathbb{Z} / q \mathbb{Z})$ - a manifestation of "strong approximation" - so that

$$
\begin{aligned}
{\left[\mathrm{SL}_{n}(\mathbb{Z}): \Gamma(q)\right] } & =\# \mathrm{SL}_{n}(\mathbb{Z} / q \mathbb{Z}) \\
& =\prod_{\substack{p \in \mathbb{P} \\
p \mid q}} \# \mathrm{SL}_{n}(\mathbb{Z} / p \mathbb{Z}) \\
& =\prod_{\substack{p \in \mathbb{P} \\
p \mid q}} \frac{\left(p^{n}-1\right)\left(p^{n}-p\right)\left(p^{n}-p^{2}\right) \cdots\left(p^{n}-p^{n-1}\right)}{p-1}
\end{aligned}
$$

where the second equality follows from the Chinese remainder theorem.

Next, the number of orbits is precisely

$$
\#\left\{g \in \mathrm{SL}_{n}(\mathbb{Z} / q \mathbb{Z}): g_{i, j}=0\right\} .
$$

By the Chinese remainder theorem we simply need to compute, for $p$ prime,

$$
\#\left\{g \in \mathrm{SL}_{n}\left(\mathbb{F}_{p}\right): g_{i, j}=0\right\} .
$$

We estimate the number of such matrices in $\mathrm{GL}_{n}\left(\mathbb{F}_{p}\right)$ and then divide by $\# \mathbb{F}_{p}^{\times}=p-1$ to get the desired count. We have $p^{n-1}-1$ choices for the $j$ th column, then $p^{n}-p$ choices for another column to be linearly independent from that column, $p^{n}-p^{2}$ choices for a third column to be linearly independent from the span of those two columns, etc.

Therefore

$$
\#\left\{g \in \mathrm{SL}_{n}\left(\mathbb{F}_{p}\right): g_{i, j}=0\right\}=\frac{\left(p^{n-1}-1\right)\left(p^{n}-p\right)\left(p^{n}-p^{2}\right) \cdots\left(p^{n}-p^{n-1}\right)}{p-1}
$$


and

$$
\#\left\{g \in \mathrm{SL}_{n}(\mathbb{Z} / q \mathbb{Z}): g_{i, j}=0\right\}=\prod_{\substack{p \in \mathbb{P} \\ p \mid q}} \frac{\left(p^{n-1}-1\right)\left(p^{n}-p\right)\left(p^{n}-p^{2}\right) \cdots\left(p^{n}-p^{n-1}\right)}{p-1} .
$$

It thus follows from (9) and (13) that

$$
\begin{aligned}
\#\left\{g \in V_{T}(\mathbb{Z}): q \mid g_{i, j}\right\} & =\frac{\#\left\{g \in \mathrm{SL}_{n}(\mathbb{Z} / q \mathbb{Z}): g_{i, j}=0\right\}}{\left[\mathrm{SL}_{n}(\mathbb{Z}): \Gamma(q)\right]} \# V_{T}(\mathbb{Z})+O\left(\prod_{\substack{p \in \mathbb{P} \\
p \mid q}} p^{n^{2}-2} \# V_{T}(\mathbb{Z})^{1-\delta}\right) \\
& =\prod_{\substack{p \in \mathbb{P} \\
p \mid q}} \frac{p^{n-1}-1}{p^{n}-1} \# V_{T}(\mathbb{Z})+O\left(q^{n^{2}-2} \# V_{T}(\mathbb{Z})^{1-\delta}\right),
\end{aligned}
$$

as claimed.

\section{SIEVING}

3.1. The Granville-Soundararajan framework. For a multiset $A=\left\{a_{1}, \ldots, a_{x}\right\}$ and a positive integer $d$, define

$$
\mathcal{A}_{d}=\#\left\{n \leq x: d \mid a_{n}\right\} .
$$

Suppose that, for square-free $d, \mathcal{A}_{d}$ can be written in the form $\frac{h(d)}{d} x+r_{d}$ for some nonnegative multiplicative function $h$ with $0 \leq h(d) \leq d$ for all square-free $d$. For a set of primes $\mathcal{P}$ and $a \in \mathcal{A}$, define $\omega_{\mathcal{P}}(a)=\#\{p \in \mathcal{P}: p \mid a\}$. Define $\mu_{\mathcal{P}}=\sum_{p \in \mathcal{P}} \frac{h(p)}{p}$ and $\sigma_{\mathcal{P}}^{2}=\sum_{p \in \mathcal{P}} \frac{h(p)}{p}\left(1-\frac{h(p)}{p}\right)$. Define $\mathcal{D}_{k}(\mathcal{P})$ to be the set of square-free integers which are the products of at most $k$ elements of $\mathcal{P}$. Finally, define $C_{k}=\frac{\Gamma(k+1)}{2^{k / 2} \Gamma\left(\frac{k}{2}+1\right)}$.

The following is [7, Proposition 3].

Claim 3.1 (Granville-Soundararajan). Uniformly for all natural numbers $k \leq \sigma_{\mathcal{P}}^{2 / 3}$, we have

$$
\sum_{a \in \mathcal{A}}\left(\omega_{\mathcal{P}}(a)-\mu_{\mathcal{P}}\right)^{k}=C_{k} x \sigma_{\mathcal{P}}^{k}\left(1+O\left(\frac{k^{3}}{\sigma_{\mathcal{P}}^{2}}\right)\right)+O\left(\mu_{\mathcal{P}}^{k} \sum_{d \in \mathcal{D}_{k}(\mathcal{P})}\left|r_{d}\right|\right)
$$

if $k$ is even and

$$
\sum_{a \in \mathcal{A}}\left(\omega_{\mathcal{P}}(a)-\mu_{\mathcal{P}}\right)^{k} \ll C_{k} x \sigma_{\mathcal{P}}^{k} \frac{k^{3 / 2}}{\sigma_{\mathcal{P}}}+\mu_{\mathcal{P}}^{k} \sum_{d \in \mathcal{D}_{k}(\mathcal{P})}\left|r_{d}\right|
$$

if $k$ is odd.

3.2. Proof of the main theorem. In this section, we prove Theorem 1.1.

Fix $n \geq 2$ and $(i, j) \in\{1, \ldots, n\}^{2}$.

Adopting the notation in [7, we define

$$
\mathcal{A}=\left\{g_{i, j}: g \in V_{T}(\mathbb{Z})\right\},
$$

a multiset of size $x:=\# V_{T}(\mathbb{Z})$.

Note that

$$
x \sim c_{n} T^{n^{2}-n}
$$

by a special case of equation (1.12) in [5, Example 1.6], with the explicit formula for $c_{n}$ given by $c_{n}=\frac{\pi^{n^{2} / 2}}{\Gamma\left(\frac{n}{2}\right) \Gamma\left(\frac{n^{2}-n+2}{2}\right) \zeta(2) \ldots \zeta(n)}$. 
For our application, we take

$$
\mathcal{P}=\left\{\text { all primes } \leq T^{\varepsilon(T)}\right\}
$$

where $\varepsilon$ is a real function which tends to 0 at infinity that we shall choose later. More precisely, we shall choose $\varepsilon(T)$ to be of the form

$$
\varepsilon(T):=\frac{1}{(\log \log T)^{\psi}},
$$

where $\psi$ is a fixed positive constant to be chosen later.

Finally, we define the multiplicative function $h: \mathbb{Z}_{\geq 1} \rightarrow \mathbb{R}_{\geq 0}$ by

$$
h(q)=\mu^{2}(q) q \prod_{\substack{p \in \mathbb{P} \\ p \mid q}} \frac{p^{n-1}-1}{p^{n}-1}
$$

In particular, for $p$ prime,

$$
\frac{h(p)}{p}=\frac{p^{n-1}-1}{p^{n}-1}=\frac{1}{p}+O\left(\frac{1}{p^{n}}\right) .
$$

Therefore, using Mertens' theorem we have

$$
\mu_{\mathcal{P}}=\log \log T+O(\log \log \log T)
$$

and likewise

$$
\sigma_{\mathcal{P}}^{2}=\log \log T+O(\log \log \log T) .
$$

Now Corollary 2.1 can be restated as follows: there exists a positive $\delta$ such that for every square-free positive integer $q$,

$$
\mathcal{A}_{q}=\frac{h(q)}{q} x+r_{q}
$$

where

$$
r_{q}=O\left(q^{n^{2}-2} x^{1-\delta}\right) .
$$

To help simplify the presentation, we introduce some probabilistic language.

For each $T>0$, we equip the finite set $V_{T}(\mathbb{Z})$ with the uniform probability measure. For the given pair $(i, j) \in\{1, \ldots, n\}^{2}$, we define the random variable $\omega_{i, j}$ on $V_{T}(\mathbb{Z})$ by $\omega_{i, j}: g \mapsto \omega\left(g_{i, j}\right)$. We can thus restate Theorem 1.1 as the convergence in distribution, as $T \rightarrow+\infty$, of the random variables $\frac{\omega_{i, j}(g)-\log \log T}{\sqrt{\log \log T}}$ to the standard Gaussian $\mathcal{N}(0,1)$.

The estimates for the moments in Claim 3.1 apply to $\omega_{\mathcal{P}}$ which is a truncated version of the random variable we are interested in; still, choosing $\varepsilon$ carefully allows us to extract all the information we need, as we make clear in the following lemma.

Lemma 3.1. As $T \rightarrow+\infty$, the random variables $\frac{\omega_{i, j}-\mu_{\mathcal{P}}}{\sigma_{P}}$ converge in distribution to $\mathcal{N}(0,1)$ if and only if the random variables $\frac{\omega_{\mathcal{P}}-\mu_{\mathcal{P}}}{\sigma_{P}} d o$.

Proof. We have

$$
c \in \mathbb{Z} \backslash\{0\}, z>1 \Rightarrow \#\{p \mid c: p>z\} \leq \frac{\log |c|}{\log z},
$$

where the primes are counted with multiplicity. Therefore if $0<|c| \leq T$ and choosing $z=T^{\varepsilon(T)}$, then

$$
\omega(c)-\omega_{\mathcal{P}}(c) \leq \frac{\log T}{\log T^{\varepsilon(T)}} \leq \frac{1}{\varepsilon(T)} .
$$

Observe that if we can choose the function $\varepsilon$ such that

$$
\frac{1}{\varepsilon(T)}=o(\sqrt{\log \log T}),
$$

then by the first sentence of [2, Remark 1], we are done. We can simply pick any $\psi \in\left(0, \frac{1}{2}\right)$ to have our truncation function $\varepsilon$ tend to 0 at infinity and satisfy (28), which completes the proof. 
Since we are interested in proving a central limit theorem, we shall not worry about the uniformity in $k$ - it is, in principle, possible to keep track of that and obtain good estimates for all moments. $k$,

In particular,Claim 3.1 reads, as $T$ (or equivalently $x$ ) goes to infinity: for every even

$$
\sum_{a \in \mathcal{A}}\left(\frac{\omega_{\mathcal{P}}(a)-\mu_{\mathcal{P}}}{\sigma_{\mathcal{P}}}\right)^{k}=C_{k} x\left(1+O\left(\frac{1}{\sigma_{\mathcal{P}}^{2}}\right)\right)+O\left(\frac{\mu_{\mathcal{P}}^{k}}{\sigma_{\mathcal{P}}^{k}} \sum_{q \in \mathcal{D}_{k}(\mathcal{P})}\left|r_{q}\right|\right)
$$

and for every odd $k$,

$$
\sum_{a \in \mathcal{A}}\left(\frac{\omega_{\mathcal{P}}(a)-\mu_{\mathcal{P}}}{\sigma_{\mathcal{P}}}\right)^{k} \ll \frac{x}{\sigma_{\mathcal{P}}}+\frac{\mu_{\mathcal{P}}^{k}}{\sigma_{\mathcal{P}}^{k}} \sum_{q \in \mathcal{D}_{k}(\mathcal{P})}\left|r_{q}\right| .
$$

To conclude using that result, we first need to show that the error term

$$
\mathcal{R}_{k}(T):=\sum_{q \in \mathcal{D}_{k}(\mathcal{P})}\left|r_{q}\right|
$$

is sufficiently small. To do so, we first observe that every $q$ in $\mathcal{D}_{k}(\mathcal{P})$ satisfies

$$
q \leq(\max \{p: p \in \mathcal{P}\})^{k} \leq T^{k \varepsilon(T)} .
$$

Using this with (27) we obtain

$$
\mathcal{R}_{k}(T) \ll x^{1-\delta} \sum_{q \leq T^{k \varepsilon(T)}} q^{n^{2}-2} \leq T^{\left(n^{2}-1\right) k \varepsilon(T)} x^{1-\delta} \ll x^{1-\delta+\frac{n^{2}-1}{n^{2}-n} k \varepsilon\left(\gamma_{n} x^{1 /\left(n^{2}-n\right)}\right)},
$$

where in the last inequality we recalled (20) and let $\gamma_{n}=\left(\frac{1}{c_{n}}\right)^{\frac{1}{n^{2}-n}}$.

Since $\varepsilon(x)=o(1)$ we obtain that

$$
\mathcal{R}_{k}(T) \ll x^{1-\frac{\delta}{2}} .
$$

Injecting the above estimate into (29) and (30) respectively, we obtain that we have recalling the main terms of (24) and (25) and noting that as $T \rightarrow+\infty, \log \log T \sim$ $\log \log x$ due to (20) - for every even $k$,

$$
\frac{1}{x} \sum_{a \in \mathcal{A}}\left(\frac{\omega_{\mathcal{P}}(a)-\mu_{\mathcal{P}}}{\sigma_{\mathcal{P}}}\right)^{k}=C_{k}\left(1+O\left(\frac{1}{\log \log x}\right)\right)+O\left((\log \log x)^{k / 2} x^{-\frac{\delta}{2}}\right)
$$

and for every odd $k$,

$$
\frac{1}{x} \sum_{a \in \mathcal{A}}\left(\frac{\omega_{\mathcal{P}}(a)-\mu_{\mathcal{P}}}{\sigma_{\mathcal{P}}}\right)^{k} \ll \frac{1}{\sqrt{\log \log x}}+(\log \log x)^{k / 2} x^{-\frac{\delta}{2}}
$$

where the implied constants depend on $k$ and we recall that $C_{k}$ is the $k$ th moment of the standard normal distribution. Equivalently, as $T \rightarrow+\infty$, we have that for every even $k$,

$$
\frac{1}{\# V_{T}(\mathbb{Z})} \sum_{a \in \mathcal{A}}\left(\frac{\omega_{\mathcal{P}}(a)-\mu_{\mathcal{P}}}{\sigma_{\mathcal{P}}}\right)^{k} \rightarrow C_{k}
$$

and for every odd $k$,

$$
\frac{1}{\# V_{T}(\mathbb{Z})} \sum_{a \in \mathcal{A}}\left(\frac{\omega_{\mathcal{P}}(a)-\mu_{\mathcal{P}}}{\sigma_{\mathcal{P}}}\right)^{k} \rightarrow 0
$$

which shows (since the normal distribution is characterised by its moments) that as $T \rightarrow+\infty$, the random variables $\frac{\omega_{\mathcal{P}}-\mu_{\mathcal{P}}}{\sigma_{\mathcal{P}}}$ converge in distribution to $\mathcal{N}(0,1)$.

Thanks to Lemma 3.1, this means that the random variables $\frac{\omega_{i, j}-\mu_{\mathcal{P}}}{\sigma_{\mathcal{P}}}$ converge in distribution to $\mathcal{N}(0,1)$. This is almost what we want, with the following lemma making the final connection and concluding the proof of Theorem 1.1. 
Lemma 3.2. As $T \rightarrow+\infty$, the random variables $\frac{\omega_{i, j}-\mu_{\mathcal{P}}}{\sigma_{\mathcal{P}}}$ converge in distribution to $\mathcal{N}(0,1)$ if and only if the random variables $\frac{\omega_{i, j}-\log \log T}{\sqrt{\log \log T}} d o$.

Proof. We write

$$
\frac{\omega_{i, j}-\log \log T}{\sqrt{\log \log T}}=\frac{\sigma_{\mathcal{P}}}{\sqrt{\log \log T}}\left(\frac{\omega_{i, j}-\mu_{\mathcal{P}}}{\sigma_{\mathcal{P}}}+\frac{\mu_{\mathcal{P}}-\log \log T}{\sigma_{\mathcal{P}}}\right)
$$

and note that, as $T \rightarrow+\infty, \frac{\sigma_{\mathcal{P}}}{\sqrt{\log \log T}} \rightarrow 1$, while $\frac{\mu_{\mathcal{P}}-\log \log T}{\sigma_{\mathcal{P}}} \rightarrow 0$, thanks to (25) and (24). The claim now follows from the third sentence in [2, Remark 1].

\section{REFERENCES}

[1] K. Alladi. An Erdős-Kac theorem for integers without large prime factors. Acta Arith, 49(1):81-105, 1987.

[2] P. Billingsley. On the central limit theorem for the prime divisor functions. Amer. Math. Monthly, 76:132-139, 1969.

[3] J. Bourgain, A. Gamburd, and P. Sarnak. Affine linear sieve, expanders, and sum-product. Invent. Math., 179(3):559-644, 2010.

[4] G. Djanković. The Erdős-Kac theorem for curvatures in integral Apollonian circle packings. Publ. Inst. Math. (Beograd) (N.S.), 89(103):11-17, 2011.

[5] W. Duke, Z. Rudnick, and P. Sarnak. Density of integer points on affine homogeneous varieties. Duke Math. J., 71(1):143-179, 1993.

[6] A. Gorodnik and A. Nevo. Quantitative ergodic theorems and their number-theoretic applications. Bull. Amer. Math. Soc. (N.S.), 52(1):65-113, 2015.

[7] A. Granville and K. Soundararajan. Sieving and the Erdôs-Kac theorem. In Equidistribution in number theory, an introduction, volume 237 of NATO Sci. Ser. II Math. Phys. Chem., pages 1527. Springer, Dordrecht, 2007.

[8] H. Halberstam. On the distribution of additive number-theoretic functions. Journal of the London Mathematical Society, s1-30(1):43-53, 1955.

[9] H. Halberstam. On the distribution of additive number-theoretic functions (ii). Journal of the London Mathematical Society, s1-31(1):1-14, 1956.

[10] D. Hensley. The number of positive integers $\leq x$ and free of prime factors $>y$. Journal of Number Theory, 21(3):286 - 298, 1985.

[11] A. Hildebrand. On the number of prime factors of integers without large prime divisors. Journal of Number Theory, 25(1):81 - 106, 1987.

School of Mathematical Sciences, Tel Aviv University, Tel Aviv, Israel

Max Planck Institute for Mathematics, Vivatsgasse 7, 53111 Bonn, Germany

E-mail address: danielelbaz88@gmail.com 